\title{
Difficult to swallow: patient preferences for alternative valproate pharmaceutical formulations
}

This article was published in the following Dove Press journal:

Patient Preference and Adherence

25 June 2009

Number of times this article has been viewed

\section{Monali Bhosle' \\ Joshua S Benner' \\ Mitch DeKoven' \\ Jeff Shelton ${ }^{2}$}

'Health Economics and Outcomes Research, IMS Health Inc, Falls Church, VA, USA; ${ }^{2}$ Answers and Insights Market Research, Inc, Indianapolis, IN, USA
Correspondence: Monali Bhosle Senior Consultant, Health Economics and Outcomes Research, IMS Health Inc., 300 N.Washington Street, Suite 303, Falls Church,VA 22046, USA

Tel + I 703-286-2890

$\mathrm{Fax}+\mathrm{I}$ 703-286-2899

Email mbhosle@us.imshealth.com
Objective: To determine the degree to which swallowing valproate (VP) tablets is an issue, the proportion of patients who would prefer an alternative formulation, and the predictors of preference.

Methods: A quantitative telephone survey of eligible adults ( $n=400, \geq 18$ years old) who currently take $(n=236)$ or previously took $(n=164)$ VP tablets within the past 6 months was conducted.

Results: More than half of the patients indicated that VP tablets were 'uncomfortable to swallow' $(68.5 \%, \mathrm{n}=274)$ and were 'very interested' $(65.8 \%, \mathrm{n}=263)$ in medications that were easier to swallow. When choosing conceptually between taking VP tablet once/day or an equally safe and effective but significantly smaller soft gel capsule twice per day, the $82.8 \%$, $(\mathrm{n}=331)$ preferred the soft gel capsule. In the multivariate regression analysis, perceiving soft gel capsules to be easier to swallow $(\mathrm{OR}=73.54 ; 95 \% \mathrm{CI}=15.01$ to 360.40$)$ and taking VP more frequently $(\mathrm{OR}=2.02 ; 95 \% \mathrm{CI}=1.13$ to 3.61$)$ were significant predictors of soft gel capsule treatment preference.

Conclusion: VP users would prefer a formulation that is easier to swallow, even if it is needed to be taken twice per day. When choosing between medications with similar efficacy and safety, physicians can consider patient preferences to optimize conditions for medication adherence.

Keywords: patient preference, valproate formulations, tablet characteristics

\section{Introduction}

Bipolar disorder, epilepsy, and migraine headache are prevalent and costly conditions for which valproate (VP) is often prescribed. Studies of US adults estimate bipolar prevalence at $3.7 \%$ to $5 \%$ with annual total cost estimates ranging from US\$10 billion to US\$45 billion. ${ }^{1-6}$ Epilepsy affects about 2.6 million Americans or just less than 1\% of the general population, producing an estimated US\$1.8 billion in direct medical costs and US\$9.3 billion in indirect costs annually (1995 US\$). ${ }^{7-9}$ Migraine headache is, by far, the most prevalent of these three disorders, affecting an estimated $17.2 \%$ of women and $6.0 \%$ of men in the US with the indirect costs to American employers estimated at approximately US\$13 billion annually. ${ }^{10-13}$

Adherence with prescribed treatment is challenging for patients with these chronic mental and neurological disorders, which all require long-term medication adherence to optimize outcomes and reduce costs. ${ }^{14-20}$ Studies find that approximately half of patients diagnosed with bipolar disorder are either partially adherent or nonadherent to pharmacological therapy. ${ }^{21-23}$ Furthermore, 30\% to $40 \%$ of bipolar patients who actively attempt to adhere to treatment are only partially successful. ${ }^{17}$ 
Estimates of treatment adherence among patients diagnosed with epilepsy were examined in observational studies finding medication noncompliance rates ranging from $59 \%$ to $71 \% .^{24,25}$ Adherence rates to pharmacologic prophylactic treatment for migraine headache was found to range from $35 \%$ to $64 \%$ in cohorts of adult Swedish migraineurs. ${ }^{26,27}$ Treatment nonadherence among patients with these conditions is likely to be a substantial contributor to unfavorable outcomes such as increased frequency of seizures ${ }^{24,25}$ and may consequently result in high direct and indirect health-related costs. ${ }^{28}$

Given the significant impact of therapeutic adherence on outcomes and costs, health care providers must recognize that the patient is the end user and ultimate decision maker when it comes to taking medications and that patient satisfaction with a medication strongly affects that decision. ${ }^{29,30}$ While medication efficacy and safety are of utmost importance, characteristics such as tablet size, shape and ease-ofswallowing can affect patients' treatment preference and adherence. ${ }^{31,32}$ A study of patients with anxiety disorder found that significantly more patients preferred the capsule form of chlordiazepoxide as compared to the tablet form. ${ }^{31}$ A 2003 representative survey of US adults $(\mathrm{N}=679)$ regarding difficulty swallowing pill-form medications found that approximately $40 \%$ of respondents had experienced difficulty swallowing pills. ${ }^{33}$ Among those having difficulty swallowing pills, the majority described feeling that it was 'stuck in the throat' $(80 \%)$, having a 'bad aftertaste in the mouth' (48\%), or 'gagging' $(32 \%) .{ }^{33}$ This study supports the assertion that difficulty swallowing pills negatively impacts medication adherence, as it found that those reporting this problem also reported delaying doses (14\%), skipping doses $(8 \%)$, and discontinuing medication $(4 \%)$ due to difficulty swallowing it. ${ }^{33}$ One in five adults surveyed reported they had hesitated to take a pill because they thought they may have trouble swallowing it, with the majority attributing this perception to pill size (84\%) and/or pill shape (29\%). ${ }^{33}$ One in 10 respondents reported choosing pills based on how difficult they might be to swallow, with women (14\%) being substantially more likely to use this criterion than men (4\%). ${ }^{33}$ Thus, patient acceptance of tablets and their adherence could be affected by size and shape of the tablet.

Valproate (VP) is commonly used to control bipolar disorder, epilepsy, and migraine prophylaxis and is most often prescribed in tablet form. The objective of the current research was to assess whether a VP formulation ('Product $\mathrm{X}$ ') with alternative soft gel characteristics such as smaller size, different composition, and different shape impact patient perceptions about ease of swallowing and treatment preference.

\section{Methods}

We conducted a quantitative telephone survey of adults $(\mathrm{N}=400, \geq 18$ years old $)$ who currently $(\mathrm{n}=236)$ or previously $(\mathrm{n}=164)$ took VP tablets $(125 \mathrm{mg}, 250 \mathrm{mg}$, or $500 \mathrm{mg}$ ) in the past 6 months. Participants were recruited using two different sources, a national on-line consumer survey panel and referrals from the National Association of the Mentally Ill. After the participant completed a short on-line survey to determine eligibility, qualified participants were invited to complete a structured interview about medication use, perceived pill characteristics, and medication preferences. A group of 14 telephone interviewers were rigorously trained to complete the survey according to nationally recognized marketing research standards. Prior to beginning the telephone interview, all participants were re-screened to ensure that they met the following eligibility criteria: $\geq 18$ years old; took or have taken VP in the past 6 months; currently take or have taken VP for at least 2 months; took or have taken $500 \mathrm{mg}$ VP tablet; have no member of the immediate household employed by or under contract for any pharmaceutical manufacturer (Figure 1). Informed consent was obtained through an online form. Respondents received an honorarium of US\$35 to US\$75 as a participation incentive (to enhance recruiting, the offered honorarium increased over time).

The survey contained 36 questions and took an average of 15 minutes to complete. The questionnaire was comprised of demographic questions, questions about perceived medication characteristics, medication use patterns, and preferences for medication characteristics (size, shape, and perceived ease of swallowing). In the univariate analyses of variance, chi-square and t-tests were used for categorical and continuous variables respectively. Multivariate logistic regression analyses were conducted to determine significant predictors of treatment preference. Variables that were statistically significant in the univariate analyses were included in the multivariate model. The dependent variable in multivariate analyses was 'Prefer Product X' ('yes' or 'no').

\section{Results}

Of 579 persons who initially responded, 400 were eligible to complete the telephone survey (Figure 1). More than half (56\%) of survey respondents were between 35 and 54 years old with only $6 \%$ being younger than 24 years old and 

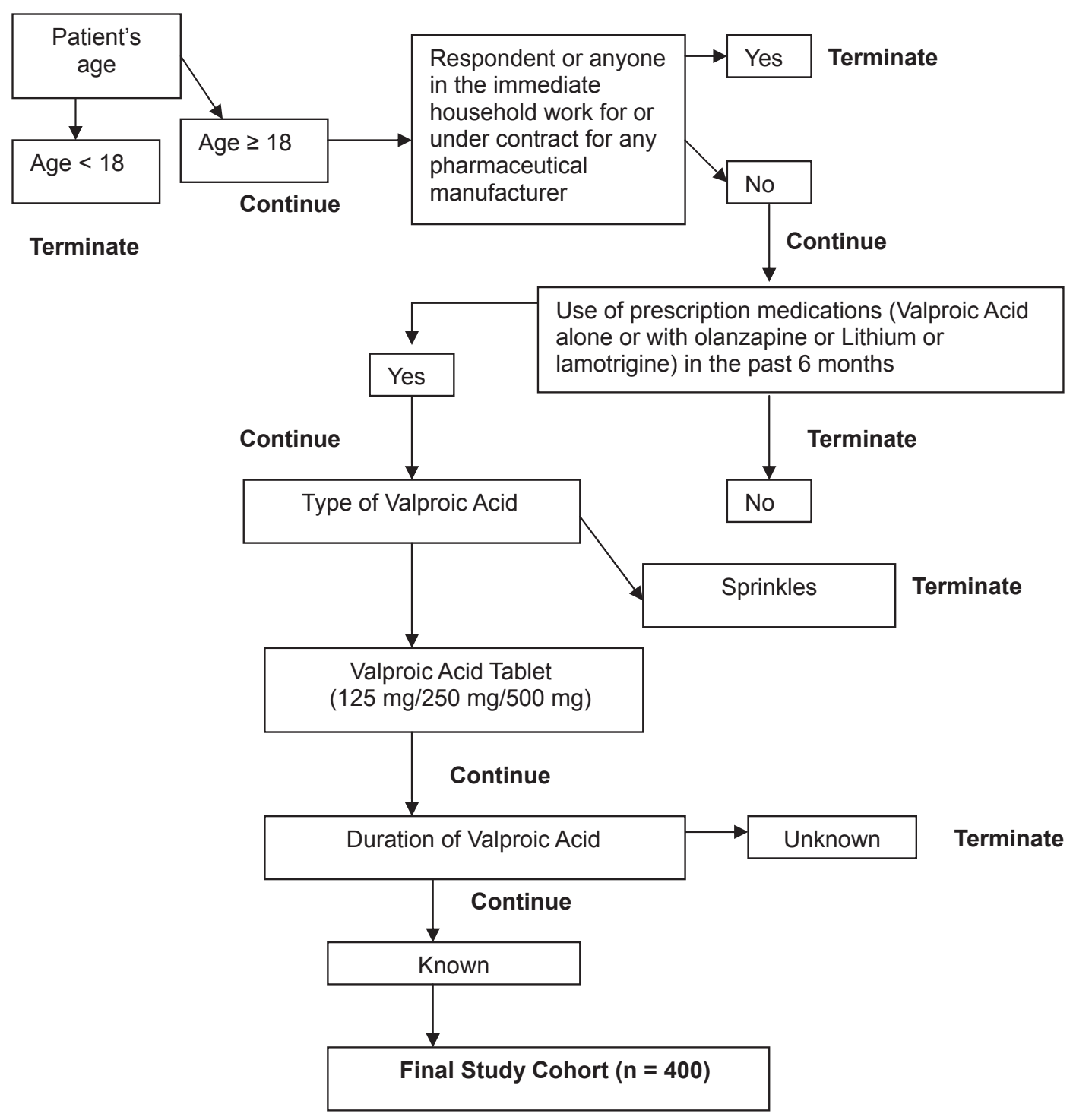

Figure I Participant screening process (initial sample size $=579$ ).

Note: From the initial pool of 579 patients, 20 patients entered the survey but did not answer any questions, hence were excluded from the study.

only $1.2 \%$ being older than 65 years. Nearly two-thirds of respondents reported having $<\mathrm{US} \$ 50,000$ in annual household income. Of the respondents, $42 \%$ were married, $32 \%$ had never been married, and $22 \%$ were divorced. Overall, respondents were well-educated, with $67 \%$ reporting they had attended some college or had either a 2-year or 4-year college degree. Most respondents also had health insurance benefits, with over $75 \%$ reporting that all or most of their treatment costs were paid for by private insurance, Medicare, or Medicaid (Table 1).

The majority of respondents were prescribed VP to control bipolar disorder $(n=260,65.0 \%)$. The remaining participants took VP primarily to prevent migraine headache $(12.5 \%$, $\mathrm{n}=50)$, control epilepsy $(11.8 \%, \mathrm{n}=47)$, or 'other conditions'
$(10.8 \%, \mathrm{n}=43)$. Respondents reported taking more than one VP tablet daily [mean (SD) $=2.5(1.2)$ and $88.0 \%$ reported taking multiple other prescription medications daily [mean $(\mathrm{SD})=5.2$ (3.6)] (Table 2)

Nearly half of the respondents discontinued taking VP as a result of the side effects they experienced $(48.5 \%, \mathrm{n}=80)$, primarily weight gain $(24.8 \%)$. In addition, slightly more than a third said that they stopped because it did not reduce their symptoms $(35.2 \%, n=58)$ (Figure 2$)$. The majority of respondents reported their VP tablet was 'uncomfortable to swallow' $(68.5 \%, \mathrm{n}=274)$ and were 'very interested' $(65.8 \%$, $\mathrm{n}=263)$ or 'somewhat interested' $(18.0 \%, \mathrm{n}=72)$ in taking a soft gel medication that appeared to be easier to swallow. When presented conceptually with a choice between taking 
Table I Respondent characteristics $(\mathrm{N}=400)$

\begin{tabular}{|c|c|c|c|}
\hline Characteristics & $\begin{array}{l}\text { Overall } \\
(N=400)\end{array}$ & $\begin{array}{l}\text { Current VP users } \\
(N=236)\end{array}$ & $\begin{array}{l}\text { Previous VP } \\
\text { users }(N=164)\end{array}$ \\
\hline \multicolumn{4}{|l|}{ Age group } \\
\hline 18-24 years & $24(6.0 \%)$ & 14 (5.9\%) & $10(6.1 \%)$ \\
\hline $25-34$ years & $84(21.0 \%)$ & $53(22.5 \%)$ & 31 (18.9\%) \\
\hline $35-44$ years & II $3(28.3 \%)$ & $64(27.1 \%)$ & 49 (29.9\%) \\
\hline $45-54$ years & III (27.7\%) & $61(25.8 \%)$ & 50 (30.5\%) \\
\hline $55-64$ years & $63(15.7 \%)$ & $39(16.6 \%)$ & $24(14.6 \%)$ \\
\hline 65 and Above & $5(1.3 \%)$ & $5(2.1 \%)$ & - \\
\hline \multicolumn{4}{|l|}{ Sex } \\
\hline Male & II 8 (29.5\%) & 92 (38.9\%) & $26(15.8 \%)$ \\
\hline Female & $282(70.5 \%)$ & $144(61.1 \%)$ & $138(84.2 \%)$ \\
\hline \multicolumn{4}{|l|}{ Marital status } \\
\hline Never married & $|2|(30.2 \%)$ & 74 (3I.4\%) & $47(28.6 \%)$ \\
\hline Married & $174(43.5 \%)$ & $104(44.1 \%)$ & 70 (42.7\%) \\
\hline Divorced & $95(23.8 \%)$ & $49(20.7 \%)$ & 46 (28.1\%) \\
\hline Widowed & $9(2.2 \%)$ & $8(3.4 \%)$ & I $(0.6 \%)$ \\
\hline Prefer not to answer & I $(0.3 \%)$ & I $(0.4 \%)$ & - \\
\hline \multicolumn{4}{|l|}{ Annual household income ${ }^{a}$} \\
\hline Less than $\$ 15,000$ & $78(19.5 \%)$ & 45 (19.1\%) & $33(20.1 \%)$ \\
\hline$\$ 15,000-\$ 29,999$ & $93(23.2 \%)$ & 44 (18.6\%) & $49(30.0 \%)$ \\
\hline$\$ 30,000-\$ 49,999$ & 77 (19.2\%) & 49 (20.8\%) & $28(17.1 \%)$ \\
\hline$\$ 50,000-\$ 69,999$ & $59(14.8 \%)$ & $34(14.4 \%)$ & $25(15.2 \%)$ \\
\hline$\$ 70,000-\$ 99,999$ & 46 (II.5\%) & $34(14.4 \%)$ & $12(7.3 \%)$ \\
\hline$\$ 100,000$ or more & $36(9.0 \%)$ & $23(9.8 \%)$ & $13(7.9 \%)$ \\
\hline Don't know/Prefer not to answer & II (2.8\%) & $7(2.9 \%)$ & $4(2.4 \%)$ \\
\hline \multicolumn{4}{|l|}{ Education } \\
\hline Some high school & $10(2.5 \%)$ & $6(2.5 \%)$ & $4(2.4 \%)$ \\
\hline High school degree or GED & $62(15.5 \%)$ & $39(16.6 \%)$ & $23(14.0 \%)$ \\
\hline Some college & $148(37.0 \%)$ & 78 (33.1\%) & $70(42.7 \%)$ \\
\hline 2- or 4-year college degree & $|2|(30.3 \%)$ & $69(29.2 \%)$ & $52(31.7 \%)$ \\
\hline Postgraduate work & $58(14.4 \%)$ & $43(18.2 \%)$ & $15(9.2 \%)$ \\
\hline Prefer not to answer & I $(0.3 \%)$ & I $(0.4 \%)$ & - \\
\hline \multicolumn{4}{|l|}{ Prescription insurance coverage } \\
\hline Pay $100 \%$ out of pocket & $28(7.0 \%)$ & $9(3.8 \%)$ & $19(11.6 \%)$ \\
\hline $\begin{array}{l}\text { Have insurance but pay significant costs } \\
\text { out of pocket }\end{array}$ & $51(12.7 \%)$ & $32(13.6 \%)$ & $19(11.6 \%)$ \\
\hline Have insurance that pays all/most of costs & 178 (44.5\%) & II $3(47.9 \%)$ & $65(39.6 \%)$ \\
\hline Have Medicare/Medicaid & $142(35.5 \%)$ & $81(34.3 \%)$ & $61(37.2 \%)$ \\
\hline Other/don't know & I (0.3\%) & $\mathrm{I}(0.4 \%)$ & - \\
\hline
\end{tabular}

aUS\$.

Abbreviation: GED, General Educational Development.

their current VP tablet once daily and taking a smaller, soft gel capsule ('Product $\mathrm{X}$ ') with equivalent safety and effectiveness twice daily, the majority $(82.8 \%, \mathrm{n}=331)$ preferred the soft gel capsule medication. An even larger majority of respondents $(85.3 \%, \mathrm{n}=341)$ indicated preferring the soft gel medication when asked, 'If both medications were available when you first began taking VP, which would you have preferred?' (Table 3)

Univariate analysis of variance revealed that respondents preferring the soft gel capsule took VP tablets more times per day $(P=0.05)$ and were significantly more likely to: perceive that soft gel capsules were easier to swallow $(P<0.001)$; 
Table 2 Valproate (VP) utilization patterns

\begin{tabular}{|c|c|c|c|}
\hline Utilization & Overall $(n=400)$ & $\begin{array}{l}\text { Current VP } \\
\text { users }(n=236)\end{array}$ & $\begin{array}{l}\text { Previous VP } \\
\text { users }(n=164)\end{array}$ \\
\hline \multicolumn{4}{|l|}{ Primary reasons for taking VP } \\
\hline Epilepsy & $47(11.7 \%)$ & $29(12.3 \%)$ & $18(10.9 \%)$ \\
\hline Bipolar disorder & $260(65.0 \%)$ & $15 \mid(63.9 \%)$ & $109(66.5 \%)$ \\
\hline Migraines & $50(12.5 \%)$ & $32(13.6 \%)$ & $18(11.0 \%)$ \\
\hline Other & $43(10.8 \%)$ & $24(10.2 \%)$ & $19(11.6 \%)$ \\
\hline \multicolumn{4}{|l|}{$\begin{array}{l}\text { Type of VP (currently using } \\
\text { or ever used) }\end{array}$} \\
\hline Tablet form, several times per day & $243(60.8 \%)$ & $133(56.4 \%)$ & $110(67.1 \%)$ \\
\hline Tablet form, once daily & $266(66.6 \%)$ & $178(75.4 \%)$ & $88(53.7 \%)$ \\
\hline \multicolumn{4}{|l|}{ Total milligrams of VP per day } \\
\hline Mean (SD) & I I 76.38 (594.59) & $1201.06(574.36)$ & I I $40.85(622.61)$ \\
\hline [Median] & {$[1000.00]$} & {$[1000.00]$} & {$[1000.00]$} \\
\hline \multicolumn{4}{|l|}{ Number of VP tablets per day } \\
\hline Mean (SD) & $2.46(1.23)$ & $2.53(1.19)$ & $2.37(1.27)$ \\
\hline [Median] & {$[2.00]$} & {$[2.00]$} & {$[2.00]$} \\
\hline \multicolumn{4}{|l|}{ Times per day taking VP } \\
\hline Mean (SD) & $1.60(0.70)$ & $1.50(0.67)$ & $1.73(0.73)$ \\
\hline [Median] & {$[1.00]$} & {$[1.00]$} & {$[2.00]$} \\
\hline \multirow{2}{*}{\multicolumn{4}{|c|}{$\begin{array}{l}\text { Concurrent use of other } \\
\text { prescription medications }\end{array}$}} \\
\hline & & & \\
\hline Yes & $352(88.0 \%)$ & $215(91.1 \%)$ & 137 (83.5\%) \\
\hline No & $48(12.0 \%)$ & $21(8.9 \%)$ & $27(16.5 \%)$ \\
\hline \multicolumn{4}{|l|}{$\begin{array}{l}\text { Total number of medications } \\
\text { (including VP) daily }\end{array}$} \\
\hline Mean (SD) & $5.24(3.63)$ & $5.58(3.68)$ & $4.7 \mathrm{I}(3.5 \mathrm{I})$ \\
\hline [Median] & {$[4.00]$} & [4.00] & [4.00] \\
\hline \multirow{2}{*}{\multicolumn{4}{|c|}{$\begin{array}{l}\text { Use of another medication } \\
\text { for condition prior to VP }\end{array}$}} \\
\hline & & & \\
\hline Yes & $266(66.5 \%)$ & I 53 (64.8\%) & 113 (68.9\%) \\
\hline No & I34 (33.5\%) & $83(35.2 \%)$ & $5 \mathrm{I}(31.1 \%)$ \\
\hline
\end{tabular}

be female $(P=0.02)$; be married $(P=0.0001)$; and have a college degree $(P=0.006)$ than those preferring VP tablets. (Table 4) In the multivariate regression analysis, perceiving soft gel capsules as being easier to swallow $(P<0.001)$, taking VP more times per day $(P=0.02)$, and being currently married $(P=0.02)$ were significant predictors of soft gel capsule treatment preference (Table 5).

Respondents preferring the soft gel capsule to the tablet formulation did not differ by age, annual household income, or prescription insurance coverage (Table 4). Those preferring soft gel capsules to tablets also did not differ by primary reason for taking VP, total mgs of VP taken per day, number of VP tablets taken per day, concurrent use of other prescription medications, or whether they had treated the primary condition with another medication prior to taking VP (Table 4).
The respondents $(n=59)$ who preferred the VP tablet even if both medications had been available when they were initially prescribed VP, were asked to give specific reasons for this preference. The most common reason given was preference for a once daily dose (34.5\%), followed by feeling that it 'works well' $(27.6 \%)$, preferring a 'name brand' $(8.6 \%)$, 'don't care' $(6.9 \%)$, 'physician's choice' (6.9\%), 'don't like gelcaps' (6.9\%), and 'side effects' (5.2\%) (Figure 3).

The respondents $(n=341)$ who indicated preferring the soft gel capsule (had it been available when they were initially prescribed VP) were also asked to give specific reasons for this preference. The most common reason given was preference for a 'smaller pill' (61.2\%), preferring an oral form that was 'easier to swallow' (55.1\%), preferring a 'soft gel' $(28.9 \%)$, feeling that it was 'faster acting' (8.8\%), feeling 




Figure 2 Reasons for discontinuing valproate (VP) $(n=164$, multiple responses were accepted; only top responses are shown).

Table 3 Respondent perceptions and treatment preference

\begin{tabular}{|c|c|c|c|}
\hline & Overall $(n=400)$ & $\begin{array}{l}\text { Current VP } \\
\text { users }(n=236)\end{array}$ & $\begin{array}{l}\text { Previous VP } \\
\text { users }(n=164)\end{array}$ \\
\hline \multicolumn{4}{|l|}{ Uncomfortable to swallow VP } \\
\hline Yes & $274(68.5 \%)$ & $162(69.6 \%)$ & $112(68.3 \%)$ \\
\hline No & $126(31.5 \%)$ & $74(31.4 \%)$ & $52(31.7 \%)$ \\
\hline \multicolumn{4}{|l|}{$\begin{array}{l}\text { Interest in taking a tablet that is } \\
\text { easier to swallow }\end{array}$} \\
\hline Very interested & $263(65.8 \%)$ & $164(69.5 \%)$ & 99 (60.4\%) \\
\hline Somewhat interested & $72(18.0 \%)$ & $4 \mathrm{I}(\mathrm{I} .4 \%)$ & $31(18.9 \%)$ \\
\hline Neither interested nor uninterested & 45 (1 I.2\%) & $22(9.3 \%)$ & $23(14.0 \%)$ \\
\hline Somewhat uninterested & $6(1.5 \%)$ & $2(0.9 \%)$ & $4(2.4 \%)$ \\
\hline Very uninterested & 14 (3.5\%) & 7 (2.9\%) & $7(4.3 \%)$ \\
\hline \multicolumn{4}{|l|}{ Appears to be easier to swallow } \\
\hline VP & $18(4.5 \%)$ & II (4.7\%) & $7(4.3 \%)$ \\
\hline Product $X$ & $382(95.5 \%)$ & 225 (95.3\%) & I 57 (95.7\%) \\
\hline \multicolumn{4}{|l|}{ Want to be made aware of } \\
\hline \multicolumn{4}{|l|}{ Product $X$ soft gel capsule } \\
\hline Yes & $312(78.0 \%)$ & $180(76.3 \%)$ & I 32 (80.5\%) \\
\hline No & $88(22.0 \%)$ & $56(23.7 \%)$ & $32(19.5 \%)$ \\
\hline \multicolumn{4}{|l|}{$\begin{array}{l}\text { Prefer if these medications were } \\
\text { equal in effectiveness and side effects }\end{array}$} \\
\hline VP & $69(17.2 \%)$ & $40(16.9 \%)$ & $29(17.7 \%)$ \\
\hline Product $X$ & $331(82.8 \%)$ & $196(83.1 \%)$ & I 35 (82.3\%) \\
\hline \multicolumn{4}{|l|}{$\begin{array}{l}\text { Prefer if these medication were } \\
\text { equal in effectiveness and side } \\
\text { effects, and comparable in costs }\end{array}$} \\
\hline VP & $63(15.87 \%)$ & $38(16.1 \%)$ & 25 (I5.2\%) \\
\hline Product $X$ & 337 (84.3\%) & $198(83.9 \%)$ & I 39 (84.8\%) \\
\hline \multicolumn{4}{|l|}{ Treatment preference $^{a}$} \\
\hline VP & 59 (I4.7\%) & $36(15.2 \%)$ & $23(14.0 \%)$ \\
\hline Product X & 34I (85.3\%) & 200 (84.8\%) & $|4|$ (86.0\%) \\
\hline
\end{tabular}

aTreatment preference was assessed using the following question: If both these medications were available when you first started taking VP, which would you have preferred your doctor to prescribe?' 
Table 4 Treatment preference (univariate analyses)

\begin{tabular}{|c|c|c|c|}
\hline \multirow[t]{2}{*}{ Variables } & \multicolumn{2}{|c|}{ Treatment preference [N (\%)] } & \multirow[t]{2}{*}{$P$-value } \\
\hline & VP $(\mathbf{N}=59)$ & Product $X(N=34 I)$ & \\
\hline \multicolumn{4}{|l|}{ VP Users } \\
\hline Current & $36(61.1 \%)$ & $200(58.6 \%)$ & \multirow[t]{2}{*}{0.73} \\
\hline Previous & $23(38.9 \%)$ & $|4|(4 \mid .4 \%)$ & \\
\hline \multicolumn{4}{|c|}{ Primary reasons for taking VP } \\
\hline Epilepsy & 7 (1 1.9\%) & $40(11.7 \%)$ & \multirow[t]{4}{*}{0.99} \\
\hline Bipolar disorder & 39 (66.1\%) & $221(64.8 \%)$ & \\
\hline Migraines & 7 (1 1.9\%) & $43(12.6 \%)$ & \\
\hline Other & $6(10.1 \%)$ & $37(10.9 \%)$ & \\
\hline \multicolumn{4}{|l|}{ Type of VP used } \\
\hline VP immediate release & $16(27.1 \%)$ & II 8 (34.6\%) & \multirow[t]{3}{*}{0.14} \\
\hline VP extended release & $30(50.9 \%)$ & 127 (37.2\%) & \\
\hline Both & I3 (22.0\%) & $96(28.2 \%)$ & \\
\hline \multicolumn{4}{|c|}{ Total milligrams of VP per day } \\
\hline Mean (SD) & I222.46 (68I.92) & $1168.40(578.90)$ & \multirow[t]{2}{*}{0.52} \\
\hline [Median] & {$[1000.00]$} & {$[1000.00]$} & \\
\hline \multicolumn{4}{|c|}{ Number of VP tablets per day } \\
\hline Mean (SD) & $2.53(1.35)$ & $2.45(1.21)$ & \multirow[t]{2}{*}{0.66} \\
\hline [Median] & {$[2.00]$} & {$[2.00]$} & \\
\hline \multicolumn{4}{|c|}{ Times per day taking VP } \\
\hline Mean (SD) & $1.44(0.68)$ & $1.62(0.70)$ & \multirow[t]{2}{*}{0.05} \\
\hline [Median] & {$[1.00]$} & {$[1.00]$} & \\
\hline \multicolumn{4}{|c|}{$\begin{array}{l}\text { Concurrent use of other } \\
\text { prescription medications }\end{array}$} \\
\hline Yes & 54 (9l.5\%) & $298(87.4 \%)$ & \multirow[t]{2}{*}{0.37} \\
\hline No & $5(8.5 \%)$ & $43(12.6 \%)$ & \\
\hline \multicolumn{4}{|c|}{$\begin{array}{l}\text { Used another medication for } \\
\text { condition prior to VP }\end{array}$} \\
\hline Yes & 39 (66.1\%) & $227(66.6 \%)$ & \multirow[t]{2}{*}{0.94} \\
\hline No & 20 (33.9\%) & II 4 (33.4\%) & \\
\hline \multicolumn{4}{|c|}{ Appears to be easier to swallow } \\
\hline VP & $16(27.1 \%)$ & $2(0.6 \%)$ & \multirow[t]{2}{*}{$<0.001$} \\
\hline Product $X$ & 43 (72.9\%) & $339(99.4 \%)$ & \\
\hline \multicolumn{4}{|l|}{ Age group } \\
\hline I8-24 years & $3(5.1 \%)$ & $21(6.2 \%)$ & \multirow[t]{6}{*}{0.92} \\
\hline $25-34$ years & $12(20.3 \%)$ & $72(21.1 \%)$ & \\
\hline $35-44$ years & 18 (30.5\%) & $95(27.9 \%)$ & \\
\hline $45-54$ years & 18 (30.5\%) & $93(27.3 \%)$ & \\
\hline $55-64$ years & $6(10.2 \%)$ & $57(16.6 \%)$ & \\
\hline$\geq 65$ years & $2(3.4 \%)$ & $3(0.9 \%)$ & \\
\hline \multicolumn{4}{|l|}{ Sex } \\
\hline Male & 25 (42.4\%) & $93(27.3 \%)$ & 0.02 \\
\hline Female & $34(57.6 \%)$ & $248(72.7 \%)$ & \\
\hline Marital status & & & \\
\hline Married & 18 (30.5\%) & $156(45.8 \%)$ & 0.0001 \\
\hline Not married & 30 (50.9\%) & $91(26.7 \%)$ & \\
\hline Divorced/Widowed & $10(16.9 \%)$ & 94 (27.5\%) & \\
\hline Prefer not to answer & I (I.7\%) & $0(0.0 \%)$ & \\
\hline
\end{tabular}


Table 4 (Continued)

\begin{tabular}{|c|c|c|c|}
\hline \multirow[t]{2}{*}{ Variables } & \multicolumn{2}{|c|}{ Treatment preference [N (\%)] } & \multirow[t]{2}{*}{$P$-value } \\
\hline & $\operatorname{VP}(\mathbf{N}=\mathbf{5 9})$ & Product $X(N=34 I)$ & \\
\hline \multicolumn{4}{|l|}{ Annual household income ${ }^{a}$} \\
\hline$<\$ 30,000$ & 27 (45.8\%) & I 44 (42.2\%) & \multirow[t]{5}{*}{0.34} \\
\hline$\$ 30,000-\$ 49,999$ & $14(23.7 \%)$ & $63(18.5 \%)$ & \\
\hline$\$ 50,000-\$ 69,999$ & $5(8.5 \%)$ & $54(15.8 \%)$ & \\
\hline$\geq \$ 70,000$ & $10(16.9 \%)$ & $72(21.1 \%)$ & \\
\hline Don't know/Prefer not to answer & $3(5.1 \%)$ & $8(2.4 \%)$ & \\
\hline \multicolumn{4}{|l|}{ Education } \\
\hline Some high school & $3(5.1 \%)$ & $7(2.1 \%)$ & \multirow[t]{5}{*}{0.006} \\
\hline High school degree or GED & $8(13.6 \%)$ & $54(15.8 \%)$ & \\
\hline 2- or 4-year college degree & $9(15.2 \%)$ & $112(32.8 \%)$ & \\
\hline Postgraduate work & $13(22.0 \%)$ & $45(13.2 \%)$ & \\
\hline Don't know/Prefer not to answer & I (I.7\%) & $0(0.0 \%)$ & \\
\hline \multicolumn{4}{|l|}{ Prescription insurance coverage } \\
\hline Pay $100 \%$ out of pocket & $3(5.1 \%)$ & $25(7.4 \%)$ & \multirow[t]{5}{*}{0.12} \\
\hline Have insurance but pay significant costs out of pocket & $5(8.5 \%)$ & $46(13.5 \%)$ & \\
\hline Have insurance that pays all/most of costs & $21(35.6 \%)$ & $157(46.0 \%)$ & \\
\hline Have Medicare/Medicaid & $30(50.8 \%)$ & $112(32.8 \%)$ & \\
\hline Other/Don't know & $0(0.0 \%)$ & $\mathrm{I}(0.3 \%)$ & \\
\hline
\end{tabular}

Notes: $P$ values in bold indicate significant at $P<0.05$.

aUS\$.

Abbreviation: GED, General Educational Development.

that it would be 'easier to digest' (7.1\%), and feeling that it would be 'easier to store' (5.3\%) (Figure 4).

\section{Discussion}

Despite the fact that tablet-related characteristics, such as difficulties in swallowing tablets due to size and shape, seem to be a well-known problem among patients (negatively affecting their treatment acceptance and preference), few studies have addressed this issue. The finding from this survey-based study indicated that the majority of patients currently or previously treated with VP tablets reported difficulty swallowing them, is of interest due to its potential usefulness in improving clinical practice and patient outcomes. Results indicate that these patients are interested in knowing about medication with similar safety and efficacy that is formulated as a smaller, soft gel capsule. Given a choice between treatment with VP tablets or smaller, soft gel capsules with similar efficacy and safety, those who perceived the soft gel capsules to be easier to swallow would prefer treatment with that medication despite having to take it twice daily.

Many patients fail to voluntarily express important treatment preferences or barriers to adherence, such as difficulty swallowing medication during the clinical encounter. Data indicate that less than a quarter of people who have difficulty swallowing their pills discuss the problem with a health professional. ${ }^{33}$ The first nationally representative survey of pill-swallowing difficulty finds that only $14 \%$ of people who have difficulty taking oral medications indicate that their health provider has brought up the topic, and only $10 \%$ report initiating conversation about this difficulty themselves. ${ }^{33}$ While there are likely to be many contributing factors to this reluctance, it has been attributed to patients' perceptions that physicians are focused on 'treating numbers' - a result of the increased emphasis on guideline-driven prescribing practices. ${ }^{34,35}$

It is well documented that patient non-adherence to medication is a substantial barrier to the effective treatment of bipolar disorder, epilepsy, and migraine prophylaxis. However, data indicate that prescribing clinicians underestimate the extent of this problem. ${ }^{22}$ One study of medication adherence in bipolar patients $(\mathrm{N}=429)$ found that although $57 \%$ reported missing all or some medication doses in the past 10 days, prescribing psychiatrists considered only $6 \%$ of these patients to be 'treatment non-adherent'. ${ }^{22}$, There is a similar tendency to overestimate patient adherence with therapy among physicians prescribing for epilepsy treatment ${ }^{36}$ and migraine prophylaxis. Thus, for these 
Table 5 Predictors of treatment preference (multivariate analysis)

\begin{tabular}{|c|c|c|c|c|c|c|}
\hline \multirow[t]{2}{*}{ Variables } & \multirow[t]{2}{*}{$\beta$ estimate } & \multirow[t]{2}{*}{ Standard error } & \multirow[t]{2}{*}{$P$-value } & \multirow[t]{2}{*}{ Odds ratio } & \multicolumn{2}{|l|}{$95 \% \mathrm{Cl}$} \\
\hline & & & & & Lower $\mathrm{Cl}$ & $\overline{\text { Higher } \mathrm{Cl}}$ \\
\hline Times per day taking VP & 0.70 & 0.30 & 0.02 & 2.02 & 1.13 & 3.61 \\
\hline \multicolumn{7}{|l|}{$\begin{array}{l}\text { Appears to be easier to } \\
\text { swallow }\end{array}$} \\
\hline Product $X$ & 4.29 & 0.81 & $<0.001$ & 73.54 & $|5.0|$ & 360.40 \\
\hline VP & - & - & - & 1.00 & - & - \\
\hline \multicolumn{7}{|l|}{ Sex } \\
\hline Female & 0.63 & 0.35 & 0.07 & 1.87 & 0.94 & 3.73 \\
\hline Male & - & - & - & 1.00 & - & - \\
\hline \multicolumn{7}{|l|}{ Marital status } \\
\hline Married & 0.94 & 0.39 & 0.02 & 2.56 & 1.19 & 5.47 \\
\hline Divorced/Widowed & 0.68 & 0.44 & 0.11 & 1.98 & 0.84 & 4.66 \\
\hline Never Married & - & - & - & 1.00 & - & - \\
\hline \multicolumn{7}{|l|}{ Education } \\
\hline High school degree or GED & 0.59 & 1.12 & 0.59 & 1.81 & 0.20 & 16.08 \\
\hline Some college & -0.18 & 1.03 & 0.86 & 0.83 & 0.11 & 6.28 \\
\hline 2- or 4-year college degree & 0.68 & 1.07 & 0.53 & 1.98 & 0.24 & 16.29 \\
\hline Postgraduate work & -0.33 & 1.06 & 0.76 & 0.72 & 0.09 & 5.76 \\
\hline Some high school & - & - & - & 1.00 & - & - \\
\hline
\end{tabular}

Abbreviations: $\mathrm{Cl}$, confidence interval; GED, General Educational Development.

disorders, medication non-adherence could be a larger problem, making it critical to recognize the problems faced by the patients while taking medications, barriers to treatment acceptance, and factors affecting patient preference.

A supportive therapeutic alliance between patients and clinicians is known to improve patient adherence with treatment in the psychiatric and neurological disorders addressed here. ${ }^{14,18,24-26,37-41}$ An important opportunity exists for physicians to build the therapeutic alliance and potentially improve treatment adherence by engaging the patient in shared decision making regarding prescription medications. ${ }^{23,25,41,42}$ Studies of other medications further support the link between medication characteristics and adherence, finding that changes in oral medication formulation affect patient adherence to prescribed therapy. ${ }^{32}$ By engaging the patient in prescribing decisions and discussing factors that may improve adherence,

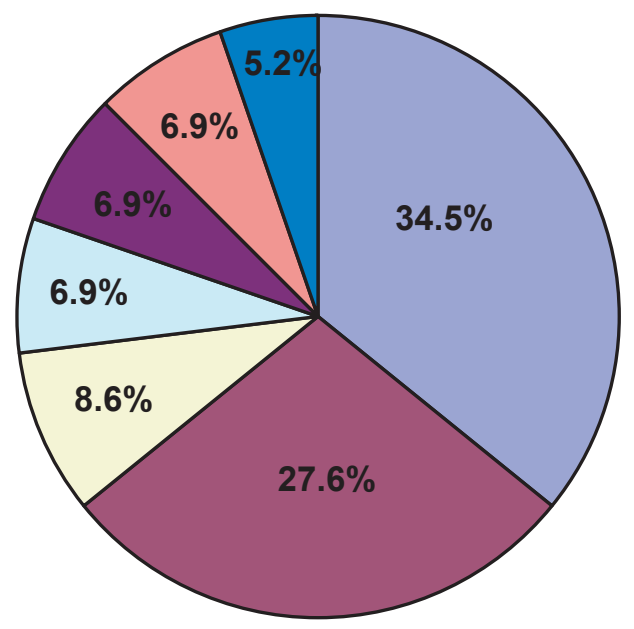

$\square$ Prefer Once Day Dose

$\square$ Works Well

$\square$ Name Brand

$\square$ Don't Care

口Physician's Choice

$\square$ Don't Like Gel Capsules

$\square$ Side Effects

Figure 3 Reasons for valproate (VP) tablet preference $(n=59)$. 


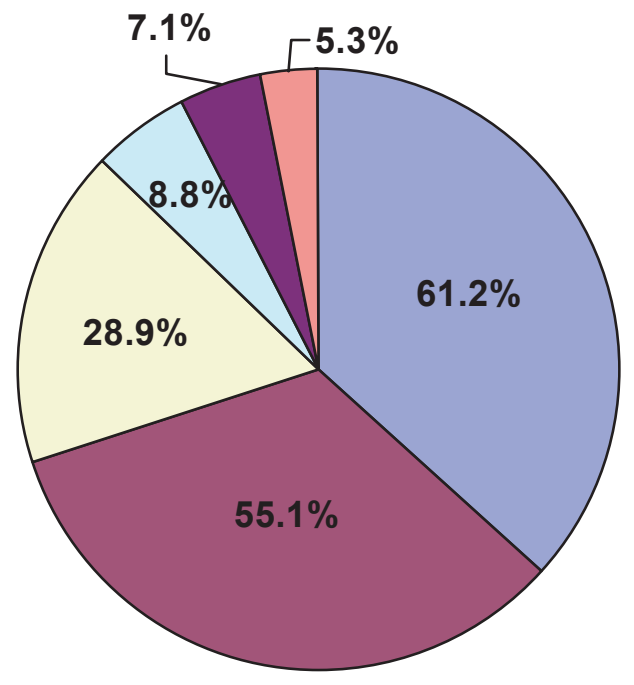

\begin{tabular}{|l|}
\hline$\square$ Smaller pill \\
$\square$ Easier to Swallow \\
$\square$ Soft Gel \\
$\square$ Faster Acting \\
$\square$ Easier to Digest \\
$\square$ Easier Storage \\
\hline
\end{tabular}

Figure 4 Reasons for Product $X$ (soft gel) preference $(n=34 I)$.

providers can optimize therapy to produce more effective management and greater improvements in the patient's condition..$^{29}$

The current study has several limitations. The US\$35 to US\$75 participation incentive that respondents received may have had effects on the results, though since it was not a focus of the study, the reasons cannot be explained. Similarly, additional analysis of marriage status, a statistically significant predictor of treatment preference, was not within the scope of the study. Most importantly, respondent demographics are not representative, thus, the results can not be generalized to other populations. It is likely that reliance on the internet for initial patient recruitment accounts for the non-representative characteristics of our respondents including high proportions of young and middle-aged persons who were college educated and had health insurance coverage for prescription medications. Time since VP cessation was not evaluated. It is possible that participants who stopped taking VP 6 months ago may have answered questions differently had they been asked nearer to when they took it.

\section{Conclusions}

The goal of any treatment is to offer an approach which eliminates or reduces the number and frequency of symptoms and produces the best possible quality-of-life while avoiding drug interactions and side effects. Given the significant impact of therapeutic adherence on clinical outcomes and health-related costs, providers must recognize the facts that patients make the ultimate treatment decision (that is to take medication as prescribed or not) and that patient satisfaction with a medication strongly affects that decision. ${ }^{29,30}$ In this study, users of VP indicated that they would prefer a formulation that is easier to swallow, even if it needed to be taken twice per day. This study provides preliminary data upon which further investigation should be based. Additional research is needed to clarify issues of patient medication preferences and to better quantify the impact of oral formulations that are easy to swallow and smaller in size on patient adherence with therapy.

\section{Acknowledgments and disclosures}

The authors thank Kat Hendrix, PhD for assistance with the manuscript preparation and review. Financial support for this study was provided by Noven Therapeutics, LLC Dr Bhosle, Dr Benner, and Mr DeKoven are employees of IMS Health, Inc. IMS Health has received compensation for research and consulting services from Noven Therapeutics, LLC Mr Shelton is an employee of Answers and Insights Market Research, Inc. Answers and Insights Market Research, Inc. received compensation from Noven Therapeutics, LLC for collecting the survey data.

\section{References}

1. Akiskal H, Bourgeois M, Angst J, Post R, Moller H, Hirschfeld R. Re-evaluating the prevalence of and diagnostic composition within the broad clinical spectrum of bipolar disorders. J Affect Disord. 2000;59 Suppl 1:S5-S30.

2. Wyatt RJ, Henter I. An economic evaluation of manic-depressive illness - 1991. Soc Psychiatry Psychiatr Epidemiol. 1995;30(5):213-219.

3. Hirschfeld R, Vornik L. Bipolar disorder - costs and comorbidity. Am J Manag Care. June 2005;(11 Suppl 3): S85-S90.

4. Begley C, Annegers J, Swann A, et al. The lifetime cost of bipolar disorder in the US: an estimate for new cases in 1998. Pharmacoeconomics. 2001;19:483-495. 
5. Sajatovic M. Bipolar disorder: disease burden. Am J Manag Care. 2005;11 Suppl 3:S80-S84.

6. Hirschfeld R, Calabrese JR, Weissman MM, et al. Screening for bipolar disorder in the community. J Clin Psychiatry. 2003;64:53-59.

7. Lesser R, Ney G. Medical management of epilepsy: issues and implications. Am J Manag Care. 1998;4 Suppl 9:S450-S452.

8. Begley CE, Famulari M, Annegers JF, et al. The cost of epilepsy in the United States: an estimate from population-based clinical and survey data. Epilepsia. 2000;41(3):342-351.

9. Annegers J. Demographics and cost of epilepsy [conference presentation]. Am J Manag Care. 1998;4(Suppl 9):S453-S457.

10. Hu XH, Markson LE, Lipton RB, Stewart WF, Berger ML. Burden of migraine in the United States: Disability and economic costs. Arch Intern Med. 1999;159:813-818.

11. Diamond S, Bigal ME, Silberstein S, Loder E, Reed M, Lipton RB. Patterns of diagnosis and acute and preventive treatment for migraine in the United States: Results from the American Migraine Prevalence and Prevention Study. Headache. 2007;47:355-363.

12. Blumenfeld A. Clinical approaches to migraine prophylaxis. Am $J$ Manag Care. 2005;11 Suppl 2:S55-S61.

13. Steiner T, Scher A, Stewart W, et al. The prevalence and disability burden of migraine in England and their relationships to age, gender and ethnicity. Cephalalgia. 2003;23:519-527.

14. Eaddy M, Grogg A, Locklear J. Assessment of compliance with antipsychotic treatment and resource utilization in a Medicaid population. Clin Ther. 2005;27:263-272.

15. Colom F, Vieta E, Tacchi M, Sanchez-Moreno J, Scott J. Identifying and improving non-adherence in bipolar disorders [Review]. Bipolar Disord. 2005;7 Suppl 5:S24-S31.

16. Scott J, Pope M. Self-reported adherence to treatment with mood stabilizers, plasma levels, and psychiatric hospitalization. Am J Psychiatry. 2002;159(11):1927-1999.

17. El-Mallakh R. Medication adherence and the use of long-acting antipsychotics in bipolar disorder. $J$ Psychiatr Pract. 2007;13(2): 79-85.

18. Buck D, Jacoby A, Baker G, Chadwick D. Factors influencing compliance with antiepileptic drug regimes. Seizure. 1997;6(2):87-93.

19. Asadi-Pooya AA. Drug compliance of children and adolescents with epilepsy. Seizure. 2005;14(6):393-395.

20. Doughty J, Baker G, Jacoby A, Lavaud V. Compliance and satisfaction with switching from an immediate-release to sustained-release formulation of valproate in people with epilepsy. Epilepsy Beh. 2003;4(6):710-716.

21. Sajatovic M, Valenstein M, Blow F, Ganoczy D, Ignacio R. Treatment adherence with antipsychotic medications in bipolar disorder. Bipolar Disord. 2006;8(3):232-241.

22. Baldessarini R, Perry R, Pike J. Factors associated with treatment nonadherence among US bipolar disorder patients. Hum Psychopharmacol. 2008;23(2):95-105

23. Scott J, Pope M. Nonadherence with mood stabilizers: prevalence and predictors. J Clinical Psychiatry. 2002;63(5):384-390.

24. Cramer JA, Glassman M, Rienzi V. The relationship between poor medication compliance and seizures. Epilepsy Behav. 2002;3(4): 338-342.
25. Jones R, Butler J, Thomas V, Peveler R, Prevett M. Adherence to treatment in patients with epilepsy: associations with seizure control and illness beliefs. Seizure. 2006;15(7):504-508.

26. Linde $\mathrm{M}$, Jonsson $\mathrm{P}$, Hedenrud $\mathrm{T}$. Influence of disease features on adherence to prophylactic migraine medication. Acta Neurol Scand. 2008;118(6):367-372.

27. Hedenrud T, Jonsson P, Linde M. Beliefs about medicines and adherence among Swedish migraineurs. Ann Pharmacother. 2008;42(1):39-45.

28. Lew K, Chang E, Rajagopalan K, Knoth R. The effect of medication adherence on health care utilization in bipolar disorder. Manag Care Interface. 2006;19(9):41-46.

29. Johnson F, Ozdemir S, Manjunath R, Hauber A, Burch S, Thompson T. Factors that affect adherence to bipolar disorder treatments: a statedpreference approach. Med Care. 2007;45(6):545-552.

30. McCracken LM, Klock PA, Mingay DJ, Asbury JK, Sinclair DM. Assessment of satisfaction with treatment for chronic pain. J Pain Symptom Manage. 1997;14(5):292-299.

31. Hussain MZ. Effect of shape of medication in treatment of anxiety states. Br J Psychiatry. 1972;120(558):507-509.

32. Ogata I, Yamasaki K, Tsuruda A, et al. Some problems for dosage form based on questionnaire surveying compliance in patients taking tamsulosin hydrochloride. Yakugaku Zasshi [Journal of the Pharmaceutical Society of Japan]. 2008;128(2):291-297.

33. Harris Interactive Inc. Pill-Swallowing Problems in America: A National Survey of Adults. New York, NY: Harris Interactive Inc. for Schwarz Pharma; 2003:1-39.

34. Institute of Medicine. Crossing the quality chasm: A New Health System for the 21st Century. Washington, DC: National Academies Press; 2001. p. 41.

35. Belcher VN, Fried TR, Agostini JV, Tinetti ME. Views of older adults on patient participation in medication-related decision making. J Gen Intern Med. 2006;21(4):298-303.

36. Dilorio C, Yeager K, Shafer P, et al. The epilepsy medication and treatment complexity index: reliability and validity testing. $J$ Neurosci Nurs. 2003;35(3):155-162.

37. Sajatovic M, Davies M, Bauer M, et al. Attitudes regarding the collaborative practice model and treatment adherence among individuals with bipolar disorder. Compr Psychiatry. 2005;46(4): 272-277.

38. Kyngas $\mathrm{H}$. Predictors of good compliance in adolescents with epilepsy. Seizure. 2001;10(8):549-553.

39. Hagell P. Compliance and noncompliance in neuroscience. $J$ Neurosci Nurs. 2000;32(3):182-184.

40. Gopinath B, Radhakrishnan K, Sarma P, Jayachandran D, Alexander A. A questionnaire survey about doctor-patient communication, compliance and locus of control among south Indian people with epilepsy. Epilepsy Res. 2000;39(1):73-82.

41. MacGregor E. The doctor and the migraine patient: improving compliance. Neurology. 1997;48 Supp1 3:S16-S20.

42. Elywn G, Edwards A, Kinnersley P. Shared decision-making in primary care: the neglected second half of the consultation. Br J Gen Pract. 1999;49:477-482.
Patient Preference and Adherence

\section{Publish your work in this journal}

Patient Preference and Adherence is an international, peer-reviewed, open access journal that focusing on the growing importance of patient preference and adherence throughout the therapeutic continuum. Patient satisfaction, acceptability, quality of life, compliance, persistence and their role in developing new therapeutic modalities and compounds to

\section{Dovepress}

optimize clinical outcomes for existing disease states are major areas of interest. This journal has been accepted for indexing on PubMed Central. The manuscript management system is completely online and includes a very quick and fair peer-review system. Visit http://www.dovepress.com/ testimonials.php to read real quotes from published authors. 